livraisons

d'Histoire

de l'Architecture

\section{Livraisons de l'histoire de l'architecture}

15 | 2008

Chambres de commerces

\title{
Une Renaissance de pierre entre Rhône et Saône : le palais du commerce de Lyon (1856-1860)
}

A Renaissance stone architecture between the Rhône and the Saône rivers: the palace of Commerce in Lyon (1856-1860)

Das architektonisches Symbol des Wiederauflebens zwischen Rhône und Saône :

der Handelspalast in Lyon

Philippe Dufieux

\section{OpenEdition}

Journals

Édition électronique

URL : http://journals.openedition.org/lha/162

DOI : $10.4000 /$ lha. 162

ISSN : 1960-5994

Éditeur

Association Livraisons d'histoire de l'architecture - LHA

Édition imprimée

Date de publication : 10 juin 2008

Pagination : 21-38

ISSN : 1627-4970

Référence électronique

Philippe Dufieux, « Une Renaissance de pierre entre Rhône et Saône : le palais du commerce de Lyon (1856-1860) », Livraisons de l'histoire de l'architecture [En ligne], 15 | 2008, mis en ligne le 10 juin 2010, consulté le 01 mai 2019. URL : http://journals.openedition.org//ha/162 ; DOI : 10.4000/lha.162

Ce document a été généré automatiquement le 1 mai 2019.

Tous droits réservés à l'Association LHA 


\title{
Une Renaissance de pierre entre Rhône et Saône : le palais du commerce de Lyon (1856-1860)
}

\author{
A Renaissance stone architecture between the Rhône and the Saône rivers: the \\ palace of Commerce in Lyon (1856-1860) \\ Das architektonisches Symbol des Wiederauflebens zwischen Rhône und Saône : \\ der Handelspalast in Lyon
}

Philippe Dufieux

\begin{abstract}
Romains, qui avez embelli la Gaule de tant de monuments, dont les seules ruines paraissent encore des merveilles ! La barbarie outrageait votre mémoire en élevant des édifices gothiques [...]. Apaisez vous, mânes augustes, ces imposantes ruines, ces superbes vestiges de votre puissance, seront désormais respectés et vont être religieusement imités. Philibert de l'Orme [...] arrête les progrès de la barbarie et régénère en France la belle architecture en la modelant sur les ruines de vos palais que le temps peine à détruire ${ }^{1}$.
\end{abstract}

Créée en 1702 puis dissoute par décret du 27 septembre 1791, la chambre de commerce de Lyon est réorganisée par arrêté du 24 décembre 1802 et n'eut jamais d'édifice approprié avant son installation au nouveau palais du commerce en 1861. Il en va de même du tribunal de commerce et du conseil des prud'hommes, créés respectivement en 1791 et en 1803. Alors que l'Hôtel-Dieu conserve invariablement sa fonction hospitalière à l'époque contemporaine, l'hôtel de ville de Lyon et l'ancien palais des Dames de Saint-Pierre, c'està-dire le palais des beaux-arts, accueillent continuellement, au cours des $\mathrm{XIX}^{\mathrm{e}}$ et $\mathrm{XX}^{\mathrm{e}}$ siècles, institutions, administrations et écoles. C'est ainsi que la chambre de commerce siège un temps à l'hôtel de ville avant d'installer ses services au palais des beaux-arts le 24 
décembre 1802. Il faudra attendre près de soixante ans pour qu'elle se dote d'un palais consulaire élevé sur les plans de René Dardel (1796-1871).

Point d'orgue des vastes transformations urbaines du Second Empire, symbole de la prospérité économique et financière de la cité rhodanienne, nulle construction n'est peut-être plus à même d'incarner la renaissance que connut la ville de Lyon au XIXe siècle que son palais du commerce, construit de 1856 à 1860. Figure majeure de ce nouvel âge d'or architectural aux côtés de Pierre Bossan (1814-1888) et d'Antoine-Marie Chenavard (1787-1883), la personnalité de René Dardel demeure aujourd'hui encore largement méconnue malgré l'ampleur de ses travaux et sa renommée contemporaine. Ce constat s'applique certes à de nombreux architectes dont les chefs-d'œuvre semblent devoir occulter durablement l'ensemble de leurs productions ${ }^{2}$. Quel rôle joue le nouveau palais du commerce dans l'émergence d'un goût néo-renaissance qui s'impose dans la seconde moitié du XIX ${ }^{e}$ siècle comme le véritable style civil lyonnais ? Quelle place occupe le palais Dardel dans la diffusion de l'architecture du $\mathrm{XVI}^{\mathrm{e}}$ siècle en France à l'époque contemporaine sur fond de spéculations historiques et archéologiques?

\section{Une carrière au service de la ville de Lyon}

Élève du lycée impérial de Lyon (1805-1813), un temps attaché à l'architecte de la ville Louis-Cécile Flachéron (1772-1835), puis élève de Joseph-Pascal Gay (1775-1832) à l'École des beaux-arts de Lyon, Dardel est engagé en avril 1814, c'est-à-dire au retour de l'Empereur de l'île d'Elbe, comme officier d'ordonnance sous les ordres du général Mouton-Duvernet, commandant de la place de Lyon. Le jeune bonapartiste vécut le désastre de 1815 et reprit ses études d'architecture la même année à Paris dans l'atelier de Jean-Nicolas Huyot (1780-1840), remplacé peu après par Charles Percier (1764-1838), avant d'intégrer la section architecture de l'École des beaux-arts de Paris. De retour à Lyon en 1822, il accompagne trois ans plus tard son confrère Claude Bonnefond (1796-1860) en Italie et rencontre à Rome Félix Duban (1798-1870) et Théodore Caruelle d'Aligny (1798-1871) avec lesquels il se lie bientôt. Selon Léon Charvet, le jeune architecte séjourne de nombreux mois en Italie, se rend à Naples, visite Paestum et Pompéi puis voyage en Sicile avant de revenir en France par Venise, Milan et Turin. Dardel fit moisson de croquis et de relevés au cours de ce séjour qui s'achève en 1827. C'est de Rome qu'il participe deux ans plus tôt au concours ouvert en vue de la restauration du Grand théâtre de Lyon dont la reconstruction fut, en définitive, confiée à Chenavard. En 1828, il participe également au concours pour la construction du nouveau palais de justice de Lyon qui sera élevé sur les plans de Pierre-Louis Baltard (1764-1846) entre 1835 et 1847. Vouant depuis la chute de l'Empire une véritable haine aux Bourbons, Dardel prend une part active à la révolution de 1830 dont les événements précipitent la chute du général Paultre de La Mothe, gouverneur de Lyon. Le 3 août 1830, le drapeau tricolore est hissé à l'hôtel de ville, Gabriel Prunelle (1777-1853) devient maire de Lyon et le $1^{\text {er }}$ juin 1831, Dardel est nommé architecte en chef en remplacement de Flachéron. Il cumule également les fonctions de voyer. Cette année-là, qui vit la première révolte des ouvriers-canuts, l'architecte s'illustre encore mais cette fois-ci comme un zélé partisan de l'ordre.

4 Avant la mise en œuvre d'une véritable politique édilitaire qui, localement, n'interviendra pas avant les années 1840, Dardel s'occupe de travaux secondaires: construction de corps de garde et de fontaines publiques, aménagement de bâtiments et travaux de restauration dont celle du palais des beaux-arts et du commerce. Depuis le 
début du $\mathrm{XIX}^{\mathrm{e}}$ siècle, le palais Saint-Pierre est transformé en une véritable cité administrative rassemblant le musée des beaux-arts (1803), l'École des beaux-arts (1807), l'université, la chambre de commerce, la Bourse ainsi que les galeries de minéralogie et de zoologie, sans oublier l'académie de Lyon et le musée des moulages « une espèce d'omnibus contenant des services commerciaux, scientifiques, industriels et artistiques » (Charvet) $)^{3}$. Dardel s'occupe en particulier de l'installation de la bibliothèque spéciale des sciences et des arts (1833), avant d'engager, entre 1840 et 1846, la restauration générale du vaste quadrilatère dont les ailes sont continuellement réaménagées dans la première moitié du siècle.

5 Parmi ses nombreux travaux édilitaires, il faut mentionner la construction du marché de la Martinière (1837), pour lequel l'architecte conçoit deux temples à colonnes doriques dans lesquels le souvenir de Paestum reste présent, l'Entrepôt des liquides de Perrache (1841) ou encore le réaménagement du Grand théâtre de Lyon (1842). Loin de s'enfermer dans la culture néoclassique, qu'il adapte avec élégance aux constructions publiques, Dardel s'intéresse très tôt à l'architecture de la Renaissance et la fontaine de la place Saint-Jean (1844) témoigne de la liberté avec laquelle l'architecte compose un monument original en s'inspirant d'un répertoire de formes dont certains détails renvoient aux décors des maisons du quartier Saint-Jean. Dardel transpose en particulier le motif supérieur du puits longtemps attribué à Delorme (Lyon, maison du Chamarier) pour composer le dais qui couronne le groupe en bronze représentant le Baptême du Christ par Jean-Marie Bonnassieux (1810-1892) ${ }^{4}$. Charvet rappelle à ce sujet combien le goût de son maitre le portait vers l'architecture française et italienne des XVI ${ }^{e}$ et $\mathrm{XVII}^{\mathrm{e}}$ siècles, admirant notamment l'art de Michel-Ange, et combien Dardel puisait dans ces modèles une inspiration féconde, ambitionnant d'harmoniser le maniérisme romain et la rigueur des ordonnances de Lescot ou de Delorme. D’ailleurs, dès la fin des années 1830, l'architecte entreprend la restauration de l'hôtel de ville de Lyon (1646-1658, Simon Maupin), du portique de la façade principale (1837) comme du beffroi (1853), mais ce dernier chantier ne sera véritablement achevé qu'entre 1854 et 1866 par son successeur Tony Desjardins (1814-1882). Aux côtés de ces chantiers, Dardel s'illustre également comme l'un des acteurs majeurs des profondes transformations qui ne devaient pas tarder à bouleverser la physionomie urbaine de la ville.

\section{Une mutation urbaine spectaculaire}

6 Au cours du XIX ${ }^{e}$ siècle, le paysage urbain de Lyon se métamorphose sous l'action conjuguée du développement de l'industrie et des affaires, du progrès économique et commercial auxquels la révolution des chemins de fer apporte son bras puissant. Longtemps resserrée dans les limites de la presqu'île, la ville s'étend progressivement au sud, au nord ainsi qu'au-delà du Rhône en direction de la plaine des Brotteaux, tandis que les faubourgs de la Croix-Rousse, de la Guillotière et de Vaise acquièrent leur physionomie industrielle et manufacturière. Avant les transformations des années 1840, le centre de Lyon conserve longtemps une physionomie héritée de l'Ancien régime notamment entre les Terreaux et Bellecour, un vaste quartier que le futur préfet de Lyon, Claude-Marius Vaïsse (1799-1864), décrit en 1853 comme « un amas ruineux de maisons d'une très grande hauteur, sans goût et sans ornement, s'élevant dans un labyrinthe de rues obscures, tortueuses, humides, privées d'air et de soleil, une ville mal bâtie, d'un abord difficile et disgracieux, inondée fréquemment $»^{5}$. Malgré des améliorations 
ponctuelles, les circulations restent problématiques et l'hygiène des rues comme celle des immeubles est absolument déplorable. Nombreux sont les contemporains à décrire le centre de Lyon comme un corps frappé de dépérissement. Il faudra attendre le mandat du maire Jean-François Terme (1840-1847) pour que la municipalité s'engage dans une véritable politique urbaine au moyen d'un plan général dressé par Dardel en 1848. Le voyer de la ville arrête un ambitieux programme de travaux comprenant à la fois la démolition de toutes les constructions établies en bordure des fleuves, l'ouverture et le prolongement de nombreuses rues, la construction de trottoirs et la réfection des chaussées comme l'installation de l'éclairage public au gaz. Mais en l'absence d'une législation adaptée, l'action des communes est longtemps réduite à des améliorations par voie d'alignement qui produisent des effets limités. C'est néanmoins dans ce cadre juridique que se réalise en 1846 la «rectification » et le prolongement de la rue Centrale (rue de Brest) qui, au moyen de démolitions ciblées, permet de relier le quartier des Terreaux à la place des Jacobins, en ouvrant une voie de circulation majeure dans le sens nord-sud, s'achevant à quelques îlots de la place Bellecour.

Dix ans plus tard, la loi sur les agglomérations du 24 mars 1852 permet à Lyon d'absorber ses faubourgs et en mars 1853, l'administration de la ville est confiée au préfet Vaïsse. Aux moyens humains et financiers s'ajoutent de nouvelles dispositions juridiques comme le décret du 25 mars 1852 qui consacre l'expropriation à grande échelle pour cause d'utilité publique. En décembre 1853, Vaïsse fait connaître les grandes lignes de son programme : « Travailler à régénérer notre vieille cité, ouvrir partout à la circulation des voies plus larges, donner aux communications des facilités, une célérité si essentielle au mouvement des affaires [...] élargir, améliorer, embellir " ${ }^{6}$. Les démolitions préalables à la percée de la rue Impériale qui doit relier, côté Rhône, la place de la Comédie à la place Bellecour, commencent l'année suivante. Le 10 décembre 1853, deux tracés pour l'ouverture de la nouvelle voie sont présentés à l'Empereur. Le projet retenu comprend notamment l'établissement d'un palais du commerce face à l'église Saint-Bonaventure aux Cordeliers et la création d'une vaste place à la hauteur de l'Hôtel-Dieu. Les travaux sont promptement menés puisque la rue Impériale est ouverte en 1857 tandis que la rue de l'Impératrice (Édouard-Herriot), qui relie la place des Terreaux à la place Bellecour, est achevée quatre ans plus tard ${ }^{7}$. Si le voyer prit une part majeure dans l'étude des projets urbains, son Plan général de la ville de Lyon dressé en novembre 1853 ne devait pourtant pas remporter l'assentiment du préfet qui n'a jamais caché sa préférence pour les ingénieurs. La mise en œuvre des grands travaux du Second Empire sera dévolue à des sociétés privées, en l'occurrence la société de la rue Impériale, ainsi qu'aux architectes Amédée Savoye (1804-1878) et Benoit Poncet (1806-1882) ${ }^{8}$ qui seront les maîtres d'œuvres de ces vastes entreprises. Le 23 mai 1853, Vaïsse demande à Dardel d'étudier un projet de palais du commerce devant comprendre une Bourse, un tribunal de commerce, le conseil des prud'hommes et enfin la condition des soies qui devait être primitivement intégrée au nouvel édifice. Alors que le projet est sur le point d'entrer dans une phase de réalisation, voici que Dardel démissionne et demande à faire valoir ses droits à la retraite à la suite d'un différent avec Vaïsse qui avait dessaisi l'architecte de sa fonction de voyer en chef pour la confier à l'ingénieur Joseph-Gustave Bonnet (1810-1875). Malgré l'insistance du préfet, Dardel refuse de reprendre ses fonctions d'architecte de la ville et fait nommer, sur sa proposition, Tony Desjardins qui entre en fonction au $1^{\text {er }}$ juillet 1854 . Soucieux de conserver la déférence de son précieux collaborateur, un mois plus tard, Vaïsse nomme 
Dardel architecte en chef du palais du commerce ${ }^{9}$. Léon Charvet, biographe de l'architecte et éminent érudit, sera son second.

\section{Le nouveau palais du commerce}

8 Le calendrier du chantier ne doit connaître aucun retard et deux années seulement séparent la présentation du programme de Vaïsse en décembre 1853 des premiers travaux de fondation du palais qui interviennent en juin $1855^{10}$. Dardel met au point les plans de l'édifice entre juillet 1854 et février 1855 , ses projets sont adoptés par la chambre de commerce en janvier 1855 ainsi que par la commission municipale le mois suivant. Dans la séance du 27 février 1854, la chambre de commerce avait déjà approuvé le principe de la réunion de différents services en un seul et même bâtiment ${ }^{11}$. En effet, la réunion des administrations municipales et préfectorales à l'hôtel de ville de Lyon sous l'égide de Vaïsse impliquait nécessairement le transfert des locaux jusqu'alors affectés au tribunal de commerce ainsi qu'au conseil des prud'hommes dans le palais communal. La pose de la première pierre a lieu le 15 mars 1856 et le palais est inauguré par l'Empereur et l'Impératrice le 25 août 1860. Un an plus tard, le tribunal de commerce, le conseil des prud'hommes, de même que les agents de change prennent possession des lieux. Les services de la chambre de commerce ne s'installeront qu'en août 1862. Si le tracé de la rue Impériale est arrêté très tôt, l'emplacement du futur palais fait l'objet de nombreuses discussions et semble avoir suscité une certaine émulation parmi les architectes lyonnais associés à la construction du nouvel axe urbain. Tous ou presque s'accordent à placer l'édifice à la hauteur de la place des Cordeliers, soit au premier tiers nord de la rue. L'emplacement choisi forme un vaste quadrilatère de $64 \times 58 \mathrm{~m}$. et offre une façade urbaine privilégiée sur la place des Cordeliers en ménageant une place publique au nord. Le choix de ne pas construire la façade principale sur la rue Impériale a été délibérément privilégié afin de permettre l'ouverture de magasins en rez-de-chaussée et ce parti se révélera en réalité une contrainte majeure dans la distribution des fonctions. L'édifice emblématique du Second Empire à Lyon se voit donc mis au service de la rue et de sa prospérité commerciale. Comment articuler la salle de la bourse et les cours intérieures dans le quadrilatère tout en assurant un éclairage suffisant notamment aux bureaux et aux pièces qui ouvrent sur l'intérieur du bâtiment? L'idée d'une vaste cour intérieure semble avoir déplu à Vaïsse qui invite son architecte à offrir à la salle de la bourse un développement plus important. C'est ce qu'il advint dans la dernière proposition de Dardel qui, en définitive, oriente la salle de la Bourse dans le sens de la longueur de l'édifice, parallèlement à la rue Impériale. Cette disposition conduit l'architecte à aménager deux cours très étroites qui permettent néanmoins d'éclairer les ailes latérales et les emplacements commerciaux du rez-de-chaussée de manière satisfaisante. La salle de la Bourse est ainsi précédée au sud, d'un grand vestibule et au nord, du salon des agents de change. Sur les façades latérales, s'ouvrent respectivement quatorze magasins orientés perpendiculairement à la salle de la Bourse. À l'étage, prennent place le tribunal de commerce, le conseil des prud'hommes ainsi que le salon de la chambre de commerce et différents dégagements. Au deuxième étage enfin, se situent le musée d'art et d'industrie et le greffe du tribunal de commerce. Les galeries longitudinales de la salle de la Bourse assurent en réalité les communications entre les massifs nord et sud du palais dont les fonctions ont été rigoureusement distinguées. Dardel place les escaliers monumentaux dans les pavillons d'angle sur la façade principale, c'est-à-dire au nord, 
tandis qu'au sud, des escaliers de service et des bureaux sont aménagés. Ce sont précisément les contraintes de circulation à l'intérieur de l'édifice qui ont invité l'architecte à dessiner deux escaliers latéraux de part et d'autre du grand vestibule, escaliers auxquels Dardel donne une ampleur majestueuse conformément à leur fonction de représentation. Il s'en expliquera à Vaïsse en janvier 1858 :

Sans prétendre discuter davantage au sujet de la longueur des escaliers de la Bourse, je crois devoir vous signaler la largeur des deux nouveaux escaliers de l'Hôtel-de-Ville de Paris qui conduisent à la grande galerie des fêtes. Ces escaliers sont absolument dans le même cas que les nôtres ; ils sont en regard et symétriques sur un même vestibule; ils ont trois mètres de largeur entre les pilastres et les colonnes en saillie [...] vous pouvez donc vous faire une idée exacte de l'effet que nous produirons ${ }^{12}$.

Dardel élabore un plan d'une rigoureuse régularité, partant d'un module de 4,5 m de côté qu'il décline invariablement selon une méthode qui rappelle immanquablement les leçons de composition de Jean-Nicolas-Louis Durand. La culture archéologique de l'architecte devait se révéler précieuse pour la conception du vaste palais qui doit matérialiser la renaissance économique et culturelle de la ville (ill. 1).

III. 1 : Lyon, palais du commerce et de l'industrie, façade principale

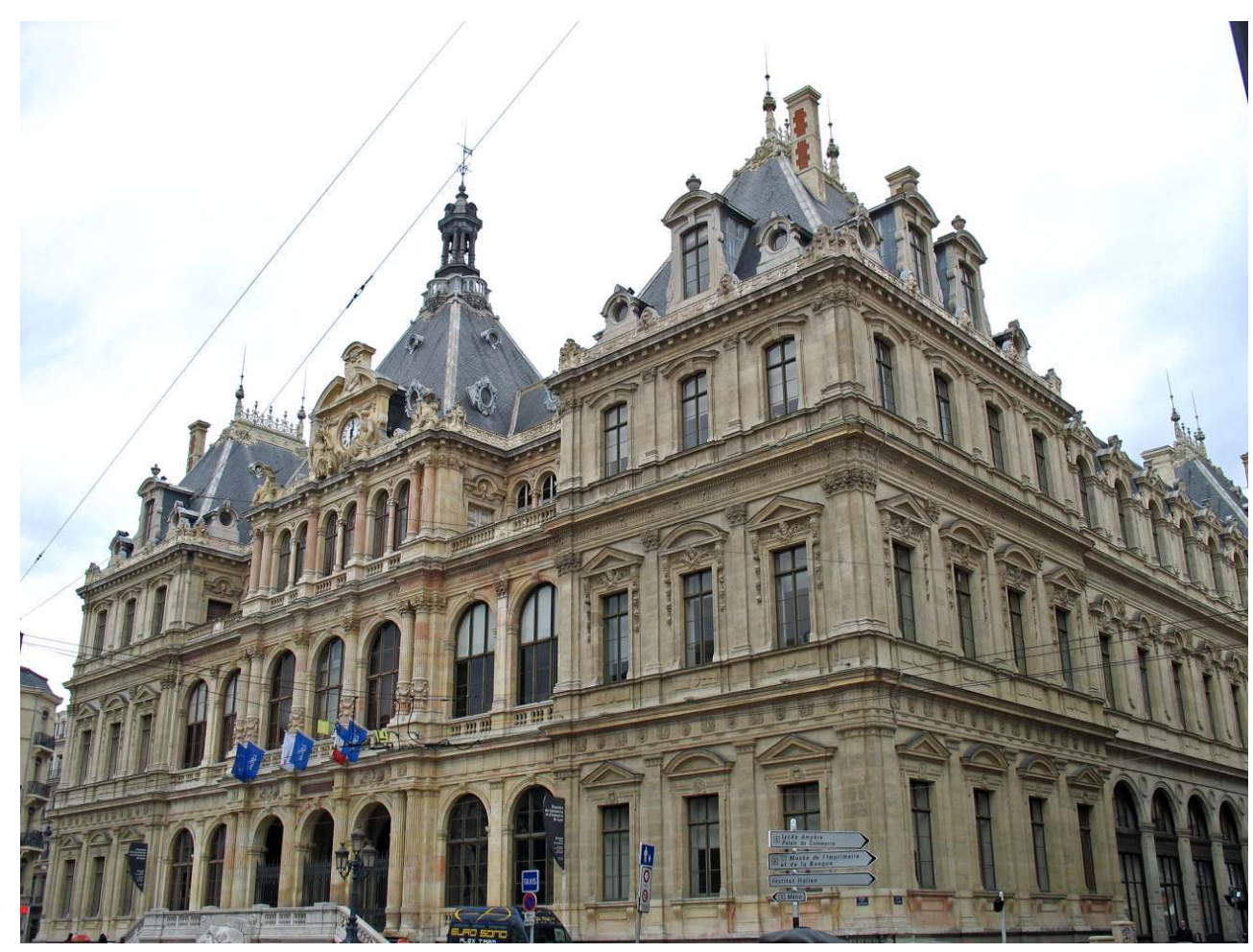

Cl. Dufieux

\section{Une construction synthétique}

Dans son courrier en date du 23 mai 1853, Vaïsse expose à Dardel le programme du futur palais et lui fait connaitre ses préférences : «Je ne voudrais pas d'architecture grecque ; elle me paraît peu en rapport avec le caractère de l'esprit général de la Ville de Lyon et je ne trouve pas que l'essai qui en a été fait pour le palais de justice soit d'un effet 
suffisamment encourageant. J'aimerais mieux quelque chose qui fût en harmonie avec l'hôtel-de-ville ou le palais des arts, qui me semblent représenter assez bien le caractère général de l'architecture lyonnaise ${ }^{13}$. » Dans ces conditions, la marge d'appréciation du conseil des bâtiments civils est très relative, ce que ne manque pas de souligner Duban, «La condition imposée par cet article supprime tout examen de principe par le Conseil sur la manière dont $\mathrm{M}$. Dardel se propose de mettre en œuvre les formes désignées par l'administration" - avant d'ajouter: "Si la richesse de l'ornementation peut paraître excessive à première vue, peut-être doit-on considérer que cet édifice est destiné à l'industrie lyonnaise, industrie de luxe et d'élégance ${ }^{14}$. " Duban émet des réserves sur l'éclairage de la salle de la Bourse qu'il juge insuffisant et se demande s'il ne serait pas préférable d'envisager une couverture métallique et des ouvertures zénithales. De même, la répétition de baies en plein cintre du corps central aux pavillons d'angles sur les façades principales lui parait peu adaptée en termes de fonctions, ces ouvertures n'ouvrant que sur des dépendances et des couloirs. Enfin, Duban attire l'attention de Dardel sur les magasins du rez-de-chaussée, qui ne doivent en aucune façon enlaidir l'édifice. Ces quelques remarques mises à part, c'est un jugement très élogieux que Duban porte sur le projet de son confrère et ami. Il faut dire que l'architecte lyonnais étudiera attentivement son projet dans un exercice archéologique savant, soucieux d'inscrire son chef-d'œuvre dans l'histoire de la ville mais plus encore de faire du palais du commerce le monument emblématique d'une nouvelle Renaissance lyonnaise qui s'affirme comme la digne héritière de l'humanisme qui s'épanouit sur les bords de Saône au XVI ${ }^{\mathrm{e}}$ siècle.

11 C'est au plan d'Ancy-le-Franc (achevé en 1550) et à travers le chef-d'œuvre de Serlio, à celui de la villa de Poggioreale (Giuliano da Majano, à partir de 1487), résidence de Charles VIII lors de la conquête de Naples (1495) et véritable modèle pour les architectes français du XVI ${ }^{e}$ siècle, que Dardel se réfère pour composer un plan-masse ponctué de pavillons d'angle peu saillants auxquels répond, aux ailes nord et sud, un pavillon central. L'architecte distingue nettement le traitement des élévations des pavillons et des ailes latérales des façades principales. De toute évidence, Dardel reproduit le dessin de la travée rythmique du premier étage de l'aile Lescot, construite au Louvre à la demande de François I ${ }^{\text {er }}$ à partir de 1546, pour dessiner les deux premiers niveaux des pavillons d'angle dans une alternance de frontons cintrés et triangulaires. Les façades latérales de l'hôtel de ville de Lyon serviront de modèle aux longues ailes du palais, sur la rue Impériale et la rue de la Bourse, auxquelles il emprunte notamment le motif de termes. De l'avis de Charvet, ces ailes forment la partie la plus faible du palais : « Il y a [...] une gêne et un manque d'unité désagréables d'aspect. Ces hautes et maigres arcades, entre lesquelles il n'a pu placer des pilastres, l'ont conduit à supprimer aussi les pilastres dans

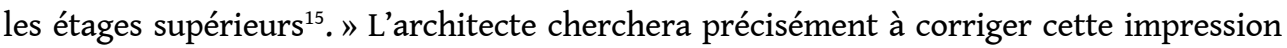
en relevant les éléments de décor, en particulier dans le traitement des fenêtres hautes dont le modèle semble s'inspirer d'Anet, mais également par la sculpture d'allégories liées au commerce et à l'économie au fronton des fenêtres du premier étage. À celles-ci, devaient s'ajouter des médaillons de Lyonnais illustres que l'architecte souhaitait voir figurer au droit des piles du rez-de-chaussée, toujours sur le modèle des façades de l'hôtel-de-ville de Lyon, mais Vaïsse s'opposa formellement à cette dernière idée. Alors que la façade nord ne s'élève que de deux niveaux, sa pendante au sud, qui en compte trois, demanda visiblement des soins attentifs pour agencer une masse composée de trois pavillons d'égale largeur et d'égale hauteur tout en relevant la partie centrale. Les galeries en plein cintre du château de Madrid, construit dans le Bois de Boulogne à partir 
de 1527 sur l'ordre de François I ${ }^{\text {er }}$ par Girolamo della Robbia et dont certaines parties ont été attribuées à Delorme, semblent avoir dicté le dessin de ces dernières façades, le vestibule ouvert côté sud abonde notamment en ce sens. L'allusion à l'entrée triomphale du Castel Nuovo de Naples (1452-1466) comme aux avant-corps de l'aile Lescot et du château d'Anet est manifeste dans la façon de dédoubler les colonnes latérales du pavillon central. L'ascendance de Delorme se traduit également dans les colonnes baguées du rezde-chaussée ainsi que celles du premier étage. Le décor de l'extrado des arcs se fait incidemment l'écho des ornements feuillagés que Delorme reproduit sur les façades d'Anet comme celle des Tuileries tandis que le visiteur est accueilli aux entrées du palais par des nymphes dont l'ascendance bellifontaine renvoie de toute évidence aux figures de Goujon à la fontaine des Innocents à Paris (1547). Craignant visiblement que la façade sud ne manque de relief, Dardel prend soin de reculer les travées du dernier étage, de part et d'autre du pavillon central afin de détacher le massif mis alors en relief par les ombres portées des pavillons. Il en va de même de la façade nord dont l'aile centrale sera privée d'un étage supplémentaire afin d'accuser plus encore l'articulation des parties. L'architecte joue habilement sur le contraste entre des pavillons et des élévations latérales minérales et les façades principales traitées à la manière de galeries largement ouvertes comme si les ordonnances françaises venaient épouser une construction antérieure dont les plans auraient été conçus par un architecte italien dans le sillage de Charles VIII, de Louis XII ou encore de François $\mathrm{I}^{\mathrm{er}}$, de retour des guerres d'Italie. Ce collage à la fois brutal et subtil a valeur de véritable métaphore d'autant qu'aux toitures brisées initialement prévues, Dardel préférera un dessin aigu afin d'accentuer un caractère "première Renaissance » dans une allusion à peine voilée à Fontainebleau ou encore à Écouen. Certains éléments du riche décor intérieur serviront cette dernière idée, comme en témoignent en particulier les balustrades à enroulements des escaliers et ceux de la salle de la bourse dont le modèle appartient au corps d'entrée d'Anet. Hormis les peintures de certains plafonds, réalisées par Alexandre Denuelle (1818-1879) et JeanBaptiste Beuchot (1821- ?) dans un style néo-renaissance, Dardel veillera à conserver aux vestibules, aux galeries couvertes ainsi qu'aux montées d'escaliers un caractère strictement minéral, soignant attentivement la stéréotomie. L'ensemble du programme décoratif accuse une ascendance néo-dix-septième siècle relevée très ponctuellement par des motifs de cuirs et des figures issues de l'imaginaire du XVI ${ }^{e}$ siècle (ill. 2). 
III. 2 : Lyon, palais du commerce et de l'industrie, escalier oriental, détail du dernier palier

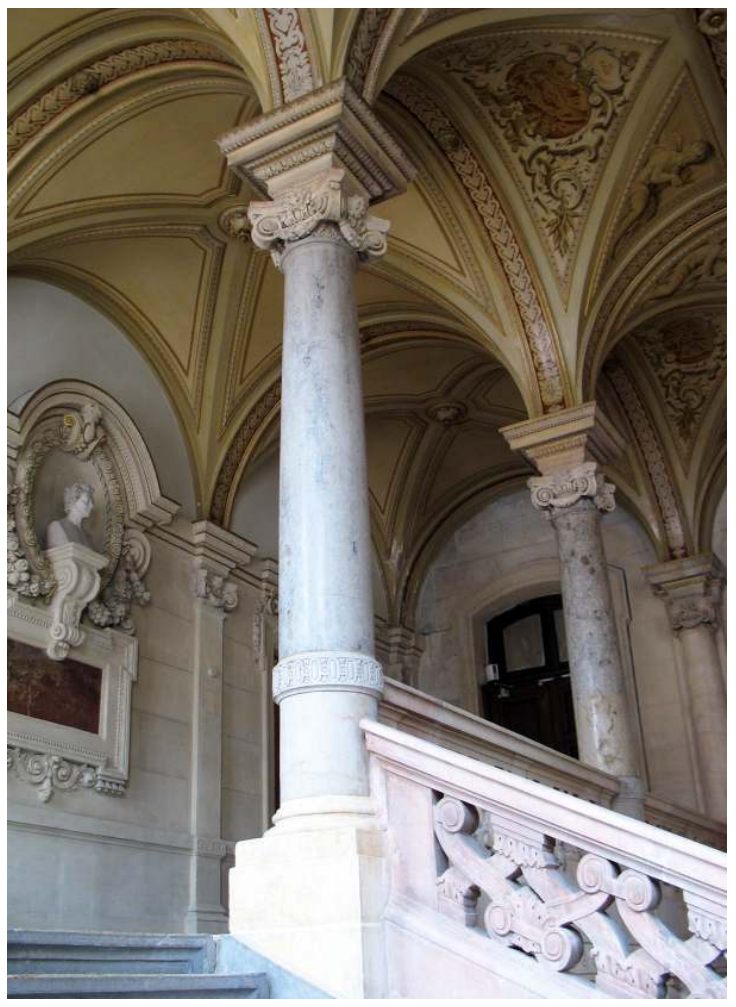

CL. DUfieuX

III. 3 : Lyon, palais du commerce et de l'industrie, la salle de la corbeille

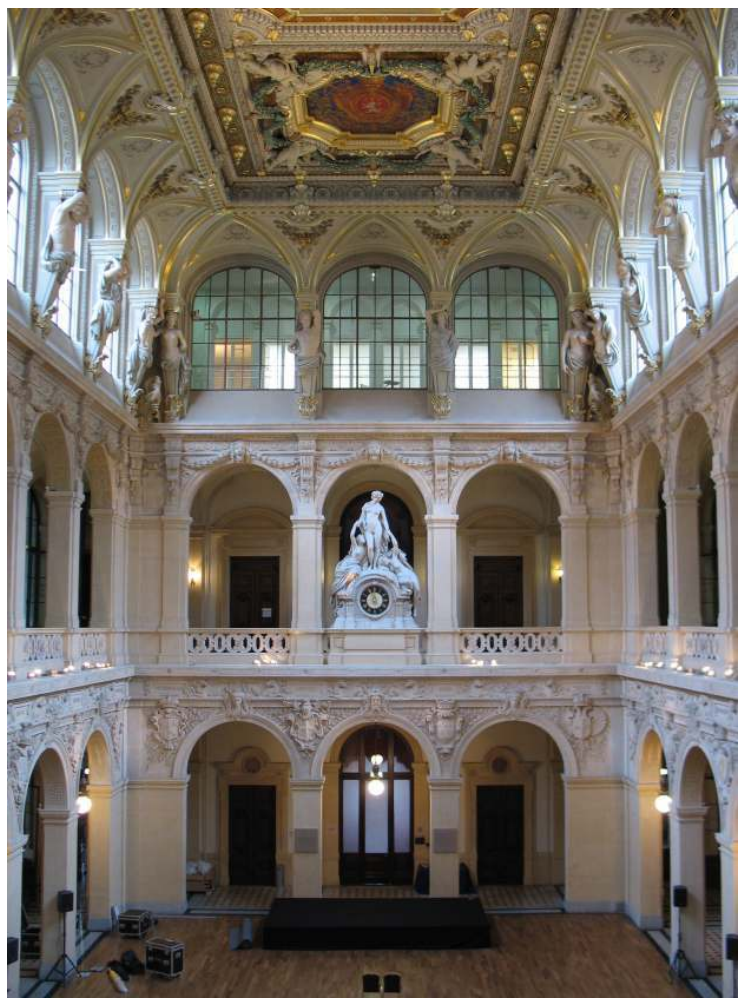

Cl. Dufieux 
La grande salle de la bourse (ill. 3) fera l'objet de tous les soins de l'architecte, recevant un important décor sculpté dont le chef-d'œuvre demeure l'horloge de Jean-Marie Bonnassieux (1810-1892) placée au premier étage côté sud dans la salle de la corbeille (1863). Si la tentation de composer une variation sur le modèle d'Anet a certainement traversé l'esprit de Dardel, on observe que celui-ci se garde de toute interprétation littérale des modèles de la Renaissance italienne et française, cherchant à produire un édifice synthétique, un rêve de pierre dans lequel les spéculations sur la gloire passée de la ville pourront désormais s'incarner et alors même que le XVI ${ }^{e}$ siècle lyonnais n'avait pas laissé d'édifice majeur dans la première capitale de la Renaissance française.

13 Le nouveau palais du commerce doit être rapproché de la bourse de Marseille, élevée par Pascal Coste et dont Dardel suit la construction ${ }^{16}$, mais également des travaux de Visconti et de Lefuel au Louvre (1853-1857) et cette remarque s'applique en particulier aux pavillons de la cour Napoléon, avec leurs colonnes détachées et leurs frontons à cariatides, qui semblent avoir inspiré l'entrée nord du palais du commerce de Lyon (ill. 4).

\section{4 : Lyon, palais du commerce et de l'industrie, entrée place de la Bourse (détail)}

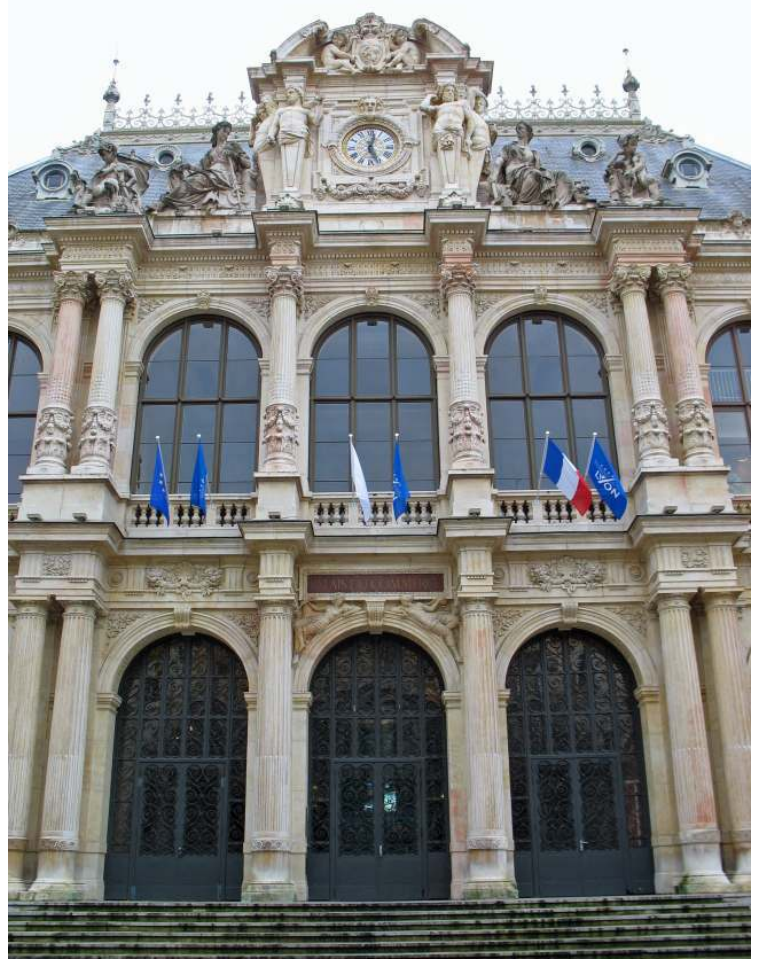

Cl. Dufieux

Comment ne pas évoquer également le nouvel Hôtel-de-Ville de Paris, incendié par la Commune en 1870 et dont la façade principale, élevée par Dominique de Cortone (à partir de 1532) a été reconstruite à l'identique entre 1872 et 1882 en style néo-Renaissance par Théodore Ballu (1817-1885) et Édouard Deperthes (1833-1898) ${ }^{17}$, tant et si bien que le lanternon qui ponctue la façade du palais du commerce de Lyon sur la place des Cordeliers accentue de fait sa parenté avec les maisons communales du XVI ${ }^{\mathrm{e}}$ siècle. Ce dernier détail mérite d'être souligné puisque la ville se voit précisément privée de ses libertés locales sous le Second Empire et que le représentant de l'État réside à l'hôtel de ville qu'il fait restaurer avec faste. Alors que les ordonnances classiques du chef-d'œuvre 
de Simon Maupin serviront le pouvoir impérial, comment ne pas voir dans l'architecture du palais du commerce de Lyon la volonté de rendre symboliquement aux édiles leurs libertés municipales en renvoyant à un $\mathrm{XVI}^{\mathrm{e}}$ siècle qui s'impose dans l'inconscient collectif lyonnais comme l'âge d'or de la ville, sur fond de prospérité économique et d'indépendance culturelle. En 1868, soit huit ans après son inauguration, Dardel fait paraître une monographie du palais du commerce accompagnée d'un descriptif historique sommaire et cette entreprise mobilisera de nombreux dessinateurs et graveurs dont Félix Barqui, Monvenoux, François Dubouchet, Rodolphe Pfnor et Louis Sauvageot pour ne citer qu'eux. Aux côtés des monographies des châteaux de Fontainebleau (1863) et d'Anet (1867) publiées par Pfnor et dont les feuilles de titre des livraisons enserrent les liasses de l'agence Dardel aux Archives municipales de $\operatorname{Lyon}^{18}$, voici que le palais du commerce se voit consacré comme un jalon majeur de l'architecture française. Alors que localement, l'art du XVI ${ }^{\mathrm{e}}$ siècle s'impose bientôt comme le véritable style lyonnais, au lendemain des événements de 1870 et en ces temps d'incertitude politique, la Renaissance française est mise à l'honneur à des fins de cohésion du corps social ; en témoigne en particulier l'architecture de la nouvelle préfecture du Rhône (1883-1890) construite par Antoine Louvier (1818-1892) dans le quartier des Brotteaux à Lyon.

\section{$L^{\prime}$ « invention » de la Renaissance française}

15 Dans le dernier quart du XIX siècle, l'architecture et les arts décoratifs de la Renaissance font l'objet d'un véritable engouement de la part des contemporains, «L'histoire de la Renaissance en France a été, jusque dans la seconde moitié de ce siècle, écrite de la façon la plus fantaisiste ", écrivait Marius Vachon en 1887, avant d'ajouter que toutes les grandes œuvres de l'art français du XVI ${ }^{\mathrm{e}}$ siècle ont été trop longtemps attribuées à des artistes italiens ${ }^{19}$. La même année, Louis Courajod inaugure son cours consacré à l'histoire de la sculpture française du Moyen Âge et de la Renaissance à l'École du Louvre en défendant l'idée de Renaissance française face à l'hégémonie artistique italienne. Quelques années plus tôt, Adolphe Berty avait ouvert la voie à cette redécouverte en publiant en 1860 Les Grands architectes de la Renaissance ${ }^{20}$ puis, peu après, La Renaissance monumentale en France ${ }^{21}$. Après le temps retrouvé des cathédrales, l'heure est en effet à l'instrumentalisation de l'art du $\mathrm{XVI}^{\mathrm{e}}$ siècle à des fins de construction de l'identité nationale. Telle est le sens de la démonstration de Pfnor, opposant le goût de Catherine de Médicis à celui de Diane de Poitiers et consacrant Anet comme le jalon fondateur de l'architecture moderne française, « Anet est une production purement française. Là, point d'alliage étrangers ${ }^{22}$, même si Delorme œuvra indifféremment pour les partis français et italien notamment pour Catherine de Médicis. On observe à ce sujet que Dardel se gardera de toutes références aux Tuileries. En tout état de cause, c'est à Delorme que revient l'honneur de refonder l'architecture française et sa ville natale ne manquera pas de s'en enorgueillir au cours du XIXe siècle. Dès 1808, l'architecte Louis Flachéron lui rend hommage dans un éloge historique qui sera couronné par l'académie de Lyon en $1814^{23}$. En 1846, la société des amis des arts met au concours un médaillon représentant les portraits de Delorme aux côtés de ceux de Coustou, Stella et Audran ${ }^{24}$. La société académique d'architecture de Lyon, fondée en 1830, ouvre à deux reprises, un concours pour un monument dédié à sa figure tutélaire, respectivement en 1856 (Fontaine avec monument à la gloire de Philibert Delorme) ainsi qu'en 1876, ce dernier monument devait être projeté face au portail Renaissance de l'église Saint-Nizier, longtemps attribué à 
l'architecte lyonnais. Dans les années 1880, les travaux se multiplient. Léon Charvet publie le premier chapitre d'une monographie restée inachevée sur Delorme avant de s'intéresser à l'œuvre qu'il a réalisée à l'abbaye de Saint-Denis ${ }^{25}$. Au lendemain de la chute du Second Empire, Delorme se retrouve sous les feux de l'actualité en raison de la démolition projetée du Palais des Tuileries, incendié en 1870, destruction qui soulève alors toutes les inquiétudes. C'est ainsi qu'en 1877, la société académique d'architecture de Lyon s'associe à la pétition ouverte par la société centrale des architectes en vue de la conservation de ces précieuses ruines. "Anéantir ce qui reste d'un monument remarquable sans chercher par tous les moyens à en faire la restauration [...] serait un véritable acte de vandalisme que rien ne pourrait justifier ", écrivait son président Louis Bresson avant d'ajouter :

Pour nous, Lyonnais, cette perte serait plus regrettable encore, et ce n'est qu'avec douleur que nous nous abandonnons à la pensée de voir compromise et vouée à la destruction la production la plus correcte de l'un de nos compatriotes. Philibert Delorme, devenu par le talent une de nos gloires nationales, était enfant de Lyon, et c'est en invoquant ce titre auprès de vous que nous espérons bien vous gagnez à la cause que nous soutenons ${ }^{26}$.

$16 \mathrm{Au}$ même moment, l'architecte Gaspard André fait figurer Delorme au panthéon artistique de la fontaine des arts élevée place des Jacobins à Lyon (1877-1886) aux côtés de Coustou, Audran et Flandrin et le monument sera précisément conçu comme un hommage en l'honneur de l'architecte lyonnais, avec ses colonnes baguées, ses cuirs stylisés, ses cartouches à l'antique et ses griffons, sans oublier sa tourelle à colonnettes qui renvoie au campanile de la chapelle d'Anet. Tenant de la main gauche le plan des Tuileries tandis que de l'autre, Delorme désigne le pavillon central du même palais, l'architecte parait conspuer avec amertume une postérité qui vient de lui ravir un nouveau chef-d'œuvre, tombé sous le coup des démolisseurs en 1883. Le seul fait que Sébastien Charléty, dans son Histoire de Lyon parue en $1903^{27}$, dénie à Delorme la paternité, pourtant remise en cause depuis longtemps, du portail de l'église Saint-Nizier agace la susceptibilité de certains architectes lyonnais si l'on en juge par la réaction de Joseph Berger qui demande à l'historien de s'expliquer preuves à l'appui ${ }^{28}$. En réalité, Delorme revêt une place majeure aux côtés de l'ornemaniste Bernard Salomon dans l'entreprise consciencieusement élaborée dans la seconde moitié du XIX ${ }^{e}$ siècle par nombre de contemporains comme Charvet, Natalis Rondot ou encore Léopold Niepce tendant à construire le mythe d'une Renaissance lyonnaise ${ }^{29}$. Alors que Fontainebleau demeure le symbole de la Renaissance italienne en France, Anet devient sous la plume d'Henri du Cleuziou le symbole de l'unité nationale, « Diane au château d'Anet se confia au génie des enfants de la patrie, au lyonnais Philibert Delorme, à Jean Goujon de Paris, à Cousin de Sens, à Germain Pilon du Mans, à Palissy de Saintes. Française, elle voulut une œuvre française ${ }^{30}$. " À l'idée d'une Renaissance italienne en France et celle d'une Renaissance française s'ajoute désormais celle d'une Renaissance lyonnaise dont le palais du commerce devient le monument emblématique en s'imposant comme un moment d'équilibre entre les cultures italienne et française. Cette construction savante se réalise certes en l'absence d'un édifice majeur dans lequel de telles spéculations auraient pu s'incarner, tant et si bien que la Renaissance à Lyon demeure un rêve de papier, à l'image des projections de Serlio ; un rêve qui ne s'est jamais traduit dans la pierre à l'exception de la galerie Bullioud de Delorme (1536), œuvre modeste à dire vrai au regard des commandes princières et royales des années 1540-1450. Dès lors, le palais Dardel devient, par métaphore, le monument « refondateur » de la première capitale de la Renaissance en 
France, véritable parangon manquant d'un XVI e siècle idéalisé qui fera le lien entre la gloire passée de la ville et ses aspirations contemporaines, entre la Renaissance et le siècle de l'industrie, deux âges d'or portés par d'ambitieuses entreprises de change et de banque, d'industrie et de commerce, dans lesquelles semble résider le génie propre de Lyon.

\section{NOTES}

1. 1. Louis Flachéron, Éloge historique de Philibert de l'Orme, architecte lyonnais, Lyon, Barret, 1814, p. 18.

2. 2. Les principales sources imprimées concernant le palais du commerce de Lyon sont les suivantes : Françoise Allain, Le Palais du commerce de Lyon, mémoire de maîtrise d'histoire de l'art sous la direction de Daniel Ternois, université Lyon 2, 1985, 3 vol ; Léon Charvet, « René Dardel (1796-1871) », Annales de la Société académique d'architecture de Lyon, t. III, 1871-1872, Lyon, Louis Perrin, 1873, p. 1-115; René Dardel, Monographie du palais du commerce élevé à Lyon sous l'administration de M. Vaïsse [...], Paris, Morel, 1868, 38 p., 48 pl.; Jean-Baptiste Monfalcon, Histoire monumentale de la ville de Lyon, Paris, Didot, 1866, t. IV, p. 40-44.

3. 3. Léon Charvet, «René Dardel (1796-1871) », Annales de la Société académique d'architecture de Lyon, op. cit., p. 35.

4. 4. Plus que la galerie Bullioud de Delorme (1536), ce dernier motif connaîtra une longue postérité à Lyon et sera souvent détourné au XIX ${ }^{\mathrm{e}}$ siècle pour servir de dais aux statues religieuses placées aux angles des immeubles. Il en va de même de la Jeanne d'Arc de Paul-Émile Millefaut à l'immeuble du Nouvelliste de Lyon, construit par Malaval (1894-1898). Philippe Dufieux, «Presse, politique et architecture. L'hôtel du Nouvelliste de Lyon ou la visibilité urbaine d'un journal de combat », Gryphe, n 16, mai 2007, p.1-9.

5. 5. [Claude-Marius Vaïsse], Rapport de monsieur le conseiller d'État chargé de l'administration du Rhône à la commission municipale sur le projet de rue impériale [...], 27 décembre 1853, Lyon, 1853, p. 9.

6. 6. Claude-Marius Vaïsse, Dix années de l'administration du département du Rhône 1852-1862, Paris, Laine et Haverd, 1862, p. 12.

7. 7. Catherine Arlaud et Dominique Bertin (dir.), De la rue impériale à la rue de la République. Archéologie, création et rénovation urbaines, Archives municipales, 1991, $150 \mathrm{p}$.

8. 8. Nizier du Puitspelu, [Clair Tisseur], Benoit Poncet et sa part dans les grands travaux publics de Lyon, Lyon, Pitrat, $1882,79 \mathrm{p}$.

9. 9. Léon Charvet, «René Dardel (1796-1871) », Annales de la Société académique d'architecture de Lyon, op. cit., p. 86-87.

10. 10. L'avant-programme est approuvé le 17 janvier 1854 par le préfet du Rhône. Si l'État participe aux dépenses induites par le percement de la rue Impériale, en revanche, Vaïsse n'obtient aucun subside de l'État ni du département pour le nouveau palais dont la construction reste à la charge de la Ville et de la chambre de commerce, soit une dépense de quatre millions de francs, valeur du terrain non comprise. Du montage financier résulte une situation complexe : la Ville de Lyon est propriétaire de l'immeuble tandis que 
la chambre de commerce en a la jouissance. L'auteur remercie Hélène Chivaley, responsable des archives de la C.C.I. de Lyon.

11. 11. Arch. de la chambre de commerce et d'industrie de Lyon, compte rendu de la séance du 27 février 1854.

12. 12. Arch. mun. Lyon, $467 \mathrm{Wp} 12$, lettre de René Dardel à Claude-Marius Vaïsse, 4 janvier 1858.

13. 13. Arch. mun. Lyon, $467 \mathrm{Wp} 12$, lettre de Claude-Marius Vaïsse à René Dardel, 23 mai 1853.

14. 14. Arch. nat., $\mathrm{F}^{21} 1861$, conseil général des bâtiments civils, rapport de Félix Duban, 5 mai 1855.

15. 15. Léon Charvet, « René Dardel (1796-1871) », Annales de la Société académique d'architecture de Lyon, op. cit., p. 93.

16. 16. Arch. mun. Lyon, 467 Wp 1 , lettre de Pascal Coste à René Dardel, 12 septembre 1858.

17. 17. Marius Vachon, Le Nouvel Hôtel-de-Ville de Paris 1872-1900, Paris, Édition du Conseil municipal,1900, IV, $322 \mathrm{p}$.

18. 18. Arch. mun. Lyon, $467 \mathrm{Wp} 12$.

19. 19. Marius Vachon, Philibert de l'Orme, Paris, J. Rouam ; Londres, Gilbert Wood \& Co , p. 3.

20. 20. Adolphe Berty, Les Grands architectes de la Renaissance: P. Lescot, Ph. De l'Orme, J. Goujon, J. Bullant, les du Cerceau, les Metezeau, les Chambiges, d'après de nombreux documents inédits des bibliothèques et des archives, Paris, Aubry, 1860, XII-172 p.

21. 21. Adolphe Berty, La Renaissance monumentale en France, Paris, 1864, 2 vol.

22. 22. Rodolphe Pfnor, Monographie du château d'Anet construit par Philibert Delorme en MDXLVIII, Paris, chez l'auteur, 1867, [avant-propos].

23. 23. Louis Flachéron, Éloge historique de Philibert de l'Orme, architecte lyonnais, Lyon, Barret, 1814, $32 \mathrm{p}$.

24. 24. Revue du Lyonnais, t. XXIV, 1846, p. 100.

25. 25. Léon Charvet, Biographie d'architectes. Philibert de l'Orme à Saint-Denis, Paris, 1891, $64 \mathrm{p}$.

26. 26. Louis Bresson, «Pétition pour la conservation du Palais des Tuileries », 5 avril 1877, Annales de la Société académique d'architecture de Lyon, t. VI, Lyon, Waltener et C $C^{\mathrm{ie}}, 1877-1880$, p. 145-148.

27. 27. Sébastien Charléty, Histoire de Lyon depuis les origines jusqu'à nos jours, Lyon, A. Rey, 310 p.

28. 28. Joseph Berger, "Rapport sur l'histoire de Lyon depuis ses origines jusqu'à nos jours publiée par Sébastien Charléty ", Annales de la Société académique d'architecture de Lyon, t. XIV, 1903-1904, Lyon, Waltener et $C^{\text {ie }}, 1905$, p. 39-41.

29. 29. Philippe Dufieux, « Des chambres de merveilles à l'université, genèse de l'histoire de l'art moderne à Lyon (1800-1914) ", dans Bicentenaire de la Société historique, archéologique et littéraire de Lyon, 28-29 septembre 2007, [actes du colloque à paraître en 2008 sous la direction de Jean-Pierre Gutton].

30. 30. Henri du Cleuziou, "Étude sur la Renaissance française à l'époque de Henri II ", dans Rodolphe Pfnor, Monographie du château d'Anet [...], op. cit., p. 43.

\section{RÉSUMÉS}

Point d'orgue des transformations urbaines du Second Empire, symbole de la prospérité économique et financière de la cité rhodanienne, nulle construction n'est peut-être plus à même d'incarner la renaissance que connut la ville de Lyon au XIX ${ }^{\mathrm{e}}$ siècle que son palais du commerce, 
construit par René Dardel (1796-1871) de 1856 à 1860. Produit d'une construction archéologique savante, le nouveau palais du commerce jouera localement un rôle majeur dans l'émergence d'un goût néo-renaissance qui s'impose dans la seconde moitié du XIX ${ }^{\mathrm{e}}$ siècle comme le véritable style civil lyonnais. Alors que le $\mathrm{XVI}^{\mathrm{e}}$ siècle n'avait pas laissé d'édifices majeurs dans la première capitale de l'humanisme en France, le nouveau palais du commerce devient dès lors le symbole de la renaissance économique et culturelle de la ville, sur fond de spéculations portant sur son âge d'or mythique.

As an acme of urban development during the Second Empire, as a symbol of economic and financial prosperity of the Rhone city, none but the palace of Commerce erected by René Dardel (1796-1871) from 1856 to 1860 may better embody the rebirth of the city of Lyon in the nineteenth century. As a result of an archeological and scholar design, the new palace of Commerce played locally a major part in initiating a Neo-Renaissance taste that would stand out as the true city style of Lyon in the second half of the nineteenth century. As no major building had been left of the sixteenth century in the first capital of humanism in France, the new palace thus was turned into the symbol of economical and cultural rebirth of the town while conjectures about its mythical golden age were debated.

Höhepunkt der urbanen Umwandlung im 2. Kaiserreich, ebenso wie Symbol für den wirtschaftlichen Wohlstand der Rhône-Stadt kann kein anderer Bau von dem Wiederaufleben der Stadt Lyon im 19.Jahrhundert besser zeugen, als der vom Architekten René Dardel (1796-1871) gebaute Handelspalast. Die von einer historischen Kennerschaft zeugenden Formen des neuen Handelskammergebäudes übten einen wesentlichen Einfluss aus auf die Neigung für den NeoRenaissancestil, der sich in der zweiten Mitte des 19. Jahrhunderts als ausgeprägter Stil der Lyoner Zivilbauten durchsetzte. Da in der ersten Hauptstadt des Humanismus kein Gebäude aus dem 16. Jahrhundert erhalten war, wurde der Handelspalast sinnbildlich für die wirtschaftliche und kulturelle Renaissance der Stadt vor dem Hintergrund der Spekulationen über deren mythisches goldenes Zeitalter

\section{AUTEUR}

\section{PHILIPPE DUFIEUX}

Philippe Dufieux est docteur de l'École pratique des hautes études (section des sciences historiques et philologiques), diplômé d'histoire, d'histoire de l'art et de sciences politiques. Il a été directeur de projets au sein de la direction de l'aménagement urbain de la Ville de Lyon. Depuis 2003, il est chargé de mission auprès du conseil d'architecture, d'urbanisme et de l'environnement du Rhône. Vice-président de la Société historique, archéologique et littéraire de Lyon, Philippe Dufieux a été chargé de cours à l'université Lyon 2. Il s'est illustré par de nombreux travaux sur l'art à Lyon aux XIX ${ }^{\mathrm{e}}$ et $\mathrm{XX}^{\mathrm{e}}$ siècles, dont Le Mythe de la primatie des Gaules. Pierre Bossan (1815-1888) et l'architecture religieuse en Lyonnais au XIX ${ }^{e}$ siècle aux Presses universitaires de Lyon (2004), Le Rêve de la maison. Cités-jardins, lotissements et habitat durable dans le Rhône (en collaboration), paru aux Éditions du CAUE du Rhône (2007) et Sculpteurs et architectes à Lyon (1910-1960) de Tony Garnier à Louis Bertola aux Éditions Mémoire active (2007). Il a participé à de nombreux colloques internationaux dont Lyon-Genève-Paris (2002), Henri Focillon (2004), Les Orientalismes en architecture (2006), Villes et religions dans l'Europe moderne et contemporaine (2006), La Colline de Chaillot et ses palais (2006) et Le Livre et l'architecte (2008). Il prépare actuellement une étude sur les élèves de Tony Garnier et l'École régionale d'architecture de Lyon ainsi qu'une monographie sur l'architecte Antoine-Marie Chenavard (1797-1883). Adresse électronique : ph.dufieux@caue69.fr 